\title{
Propuesta didáctica para una primera aproximación a la escritura del artículo científico en Euskera
}

\begin{abstract}
RESUMEN
Producir textos académicos escritos es una de las primeras tareas a las que se enfrenta el alumnado una vez ingresa en la universidad. Iniciar los estudios superiores trae consigo la necesidad de aprender las formas de interacción oral y escrita de esa nueva comunidad discursiva y, como consecuencia, entrar en un proceso de alfabetización académica. Escribir será, además, la herramienta con la que los estudiantes mostrarán los conocimientos aprendidos y el medio por el que serán evaluados. Este trabajo presenta una herramienta didáctica para que el alumnado se acerque, con la ayuda del profesorado o de manera autónoma, a la escritura de textos académicos en euskera, independientemente del área en el que estén recibiendo su formación. Se trata de una propuesta didáctica que busca mejorar la formación en Educación Superior y responder a las demandas educativas mediante el enfoque basado en los géneros discursivos. En este caso, el género seleccionado es el artículo científico. La aplicación de esta propuesta contribuye a identificar las características de estos textos académicos y a familiarizarse con las demandas comunicativas de estas producciones. De manera paralela, propicia el aprendizaje autónomo y el desarrollo de habilidades discursivas para desenvolverse en la comunidad universitaria.
\end{abstract}

Palabras clave: Alfabetización académica; Géneros discursivos; Herramienta didáctica; Enseñanza Superior.

\section{INTRODUCCIÓN}

Acceder a la Educación Superior es sinónimo de entrar a formar parte de una nueva comunidad discursiva en la que estudiantes, docentes e investigadores interactúan a través de textos orales y escritos cuyo fin último es la difusión del saber. Como consecuencia, el alumnado se ve en la necesidad de adquirir los conocimientos necesarios para comunicarse en este medio o, lo que es lo mismo, sumergirse en un proceso de alfabetización o enculturación académica (Lea \& Street, 2006; Prior \& Bilbro, 2012). En la actualidad, conscientes de la dificultad que supone para el estudiante desarrollar de manera autónoma
Mari Mar Boillos Pereira ${ }^{i}$ Universidad del País Vasco/Euskal Herriko Unibertsitatea, España

Garbiñe Bereziartua Etxeberria ${ }^{i i}$ Universidad del País Vasco/Euskal Herriko Unibertsitatea, España 
las habilidades orales y escritas, cada vez son más los grados y posgrados que ofrecen formación específica en esta materia. Sin embargo, el profesorado no tiene, en ocasiones, recursos para poder formar a sus estudiantes en el discurso académico propio de su área del saber o carece de los artefactos pedagógicos para dar respuesta a las demandas del aula (Carlino et al., 2013; Guzmán-Simón \& García-Jiménez, 2017).

En este contexto, el objetivo de este trabajo es presentar una herramienta didáctica para la formación en escritura académica en los primeros estadios de la formación universitaria. Este material tiene base en los principios del enfoque basado en los géneros. Concretamente, se construye sobre las premisas de la Lingüística sistémico-funcional y los planteamientos metodológicos de la Escuela de Sydney (Rose \& Martín, 2012). Así mismo, parte de la idea de la importancia de ofrecer herramientas al alumnado para que puedan desarrollar, de manera autónoma, su capacidad de expresión dentro de la comunidad académica. Por último, y no por ello menos importante, se trata de una propuesta que aborda la enseñanza de la escritura académica en euskera, lengua en la que los recursos y materiales con esta finalidad están en un primer estadio de desarrollo.

Así, este artículo ofrecerá, en primer lugar, una reflexión acerca de los retos que supone el proceso de alfabetización académica, especialmente en las primeras etapas de la formación universitaria. A continuación, se describirá la metodología de enseñanza seleccionada para esta propuesta. Finalmente, se presentará una descripción detallada del contexto de implantación de esta propuesta, la naturaleza de esta, y se comentarán, a modo de ejemplo, algunos de los ejercicios de los que forma parte este material. Por último, se reflexionará acerca de los beneficios que conlleva.

\section{MARCO TEÓRICO}

\subsection{LA ALFABETIZACIÓN ACADÉMICA}

Hablar de alfabetización académica supone hablar de uno de los retos a los que se enfrentan los estudiantes una vez se integran en el contexto universitario. A pesar de que los estudiantes, en las etapas educativas precedentes, han sido capaces de desarrollar su competencia comunicativa en las lenguas oficiales e, incluso, en otras lenguas, al llegar a esta etapa formativa se ven en la necesidad de interiorizar las formas propias de comunicación de la nueva comunidad discursiva de la que van a formar parte. Es decir, no solo van a estar expuestos a discursos orales y escritos propios de la disciplina académica en la que se están formando, sino que van a tener que adquirir la habilidad para producirlos (Carlino, 2005).

La revisión llevada a cabo por Carlino (2013) sobre los estudios acerca de este tema muestra acuerdo al afirmar que el desarrollo de la competencia comunicativa es un aprendizaje que se desarrolla a lo largo de la vida. Al llegar a la universidad, se observa que algunos estudiantes arrastran carencias a nivel de discurso de etapas educativas anteriores que, de estar fosilizadas, pueden perjudicar el desarrollo comunicativo en este nuevo contex- 
to. De ahí la necesidad de que, entre las competencias que deben tratarse en el contexto universitario, esté también la competencia comunicativa académica.

Estas afirmaciones van en consonancia con los planteamientos educativos que se están promoviendo a nivel europeo. De acuerdo con las premisas establecidas en el Espacio Europeo de Educación Superior, ser capaz de desenvolverse en el ámbito profesional implica adquirir la competencia para interactuar, de manera oral y escrita, dentro de esta área del saber y dentro de los géneros discursivos que se producen en dicho contexto (Ezeiza, 2008).

Según la corriente de la que se trate, la definición, la categorización y la caracterización sociolingüística de los géneros discursivos puede variar significativamente. En este trabajo, se entienden por género discursivo académico todos aquellos géneros que se llevan a cabo en las distintas áreas del saber: resúmenes, actas, reseñas, etc. Cada uno de ellos con sus particularidades en cuanto a apartados, estilos, extensión y objetivos, entre otros (Russell \& Cortes, 2012). Estos géneros, además, poseen particularidades que vienen determinadas por la lengua en la que se producen. Como consecuencia, no solo se trata de conocer los géneros discursivos, sino también las singularidades que dichos géneros discursivos poseen en las diferentes lenguas en las que va a interactuar el estudiante (Aguilar, 2017).

Para entender mejor esta idea, es necesario retrotraerse al origen del concepto de género discursivo que tuvo como precursor a Bajtín (1979, Capítulo 4). En sus palabras, los géneros son aquellos grupos de texto que se producen en un contexto determinado y cuyas características son identificables por los hablantes de una comunidad discursiva. Es decir, formas de discurso estereotipadas que tienen una naturaleza relativamente estable. Swales (1996) diferenció los géneros de las tipologías en tanto que todos los textos de un mismo género persiguen el mismo objetivo y tienen unos contenidos, estructuras y estilos que son identificables por todos los miembros de una comunidad discursiva. Mientras tanto, las tipologías son formas estables y clasificadas, según Werlich (1975), en cinco categorías (descriptivo, narrativo, expositivo, argumentativo y directivo) que pueden convivir dentro de un mismo género.

En la esencia de los textos académicos, orales y escritos, está también el proceso que subyace a la producción de estos. Castelló (2009) distribuye en tres grupos las demandas que exige la producción de textos científicos. En el primero de ellos, se destacan las decisiones que tienen que ver con la identificación de las variables que definen la situación de comunicación en la que se produce el texto: objetivo, receptores, conocimientos previos del tema, el canal, etc. En segundo lugar, quien produzca el texto tendrá que controlar las diversas tareas que requiere el proceso de composición: búsqueda de información, lectura, reorganización de ideas, revisión de las ideas seleccionadas, etc. En tercer y último lugar, se sitúan las demandas que derivan del área de conocimiento en el que se produce el texto: autores más relevantes, líneas de investigación prioritarias, teorías, etc. El texto, en definitiva, se sitúa en un contexto social e histórico determinado y tiene que gestarse en armonía con el resto de los textos que ya están en circulación. 
En consecuencia, producir un texto académico no es trasladar unas ideas de manera automática, sino poner en marcha diversas estrategias cognitivas que también requieren de formación y entrenamiento. Viene a colación, por tanto, la idea de Scardamalia y Bereiter (1992) según la cual, en el caso de la escritura, existe una diferencia entre escritores expertos y neófitos. En el primer grupo se integrarían las personas capaces de poner en marcha los diversos procesos cognitivos que implica la escritura, tales como la interpretación de ideas, la reformulación o la confrontación de teorías, entre otras. En el lado opuesto, los neófitos reproducen de manera directa y sin interpretación la información, sin que haya una planificación previa y sin conciencia de las particularidades en las que se produce la situación de comunicación.

En este sentido, también parece de interés destacar que cuanto mayor sea la madurez comunicativa del estudiante una vez llega a la universidad, mayor será también su capacidad para desenvolverse en el nuevo contexto discursivo. No obstante, será necesario interiorizar los esquemas de pensamiento, la terminología, las formas de escritura, etc., del área del saber en el que se esté formando para poder hacer un uso estratégico de todos esos saberes previos que se han mencionado (Boillos, 2018).

Además, la escritura tiene un valor añadido en tanto que escribir posibilita los conceptos e ideas asociados a un tema determinado. Es decir, cuando el alumnado produce un texto académico, el valor epistémico de la escritura conlleva que se interioricen los planteamientos, se contrasten puntos de vista y se generen nuevas ideas asociadas a las que se están aprendiendo (Capomagi, 2013).

En definitiva, todas las ideas expuestas llevan a subrayar la necesidad de tratar el proceso de alfabetización académica desde el primer año en el que el alumnado ingresa en la comunidad universitaria. Una progresiva aproximación a los textos, familiarizarse con su complejidad, entrenar las características de cada género (contextos en los que se producen, extensión, objetivos, etc.) ayudará al estudiante a afrontar, de manera paulatina, retos de mayor complejidad. Podría decirse que todo ello supondrá desarrollar su competencia comunicativa académica para ser capaz de llevar a cabo la tarea final de los grados universitarios: la escritura del Trabajo de Fin de Grado y su defensa ante un tribunal.

El interrogante surge al reflexionar acerca de quién tiene que ser el agente o los agentes encargados de llevar a cabo la alfabetización académica. Según Carlino $(2002,2005)$, esta formación debería realizarse dentro de las asignaturas. Y es que, si bien es cierto que las asignaturas orientadas específicamente a este fin son clave para que el alumnado identifique las características de los discursos académicos, trabajar esta competencia dentro de las demás asignaturas permite que el proceso de adquisición tenga lugar dentro de una situación de comunicación en el que se da respuesta a demandas reales propias del ámbito universitario. Esta idea viene reforzada por el hecho de que, en algunos casos, el éxito de un aprendiz no tiene que ver con su capacidad para entender los conceptos de una asignatura, sino que viene determinado por su incapacidad para expresar de manera oral o escrita las ideas que se han aprendido (Carlino \& Martínez, 2009). 


\subsection{ENSEÑAR A TRAVÉS DE LOS GÉNEROS}

Tal y como se ha venido adelantando, parece necesario abordar la formación en alfabetización académica desde los primeros estadios de la Educación Superior. Entre las diferentes herramientas de las que puede disponer el docente encargado de este reto, la enseñanza a través de los géneros surge como una herramienta que permite dar respuesta a las demandas de la comunidad universitaria. A continuación, se exponen los principios básicos de este enfoque, así como sus premisas metodológicas.

El interés por la enseñanza a través de los géneros creció significativamente a finales del siglo XX y tuvo como resultado el surgimiento de diferentes teorías y líneas de trabajo. Entre ellas, podrían identificarse dos como las más relevantes: la Escuela de Sydney, que emerge en los principios de la Lingüística sistémico-funcional, y la Escuela de Ginebra, con base en el interaccionismo sociodiscursivo. En este trabajo se pone el foco en la primera de ellas, la Escuela de Sydney, que recibió ese nombre por tener uno de los ejes de expansión en Australia a partir de los años 80 (Rose, 2015, 2017; Rose \& Martín, 2012). Este enfoque parte de la idea de que una metodología de enseñanza de las lenguas basada en los géneros permite conocer las características discursivas de los textos que se producen en una comunidad y en un contexto determinados con un objetivo de comunicación concreto.

Martin (1999) establece que el proceso de enseñanza debe construirse en tres fases fundamentales. La primera, la fase del modelaje, se centra en el análisis de los géneros por parte de alumnado y profesorado para la identificación de las características lingüísticas, estructurales y comunicativas. En la segunda de las fases, conocida como construcción, el alumnado se ejercita mediante la modificación, manipulación, etc., de textos. Por último, se da paso a la construcción independiente, fase en la que el alumnado tiene que producir un determinado género a partir de los conocimientos que ha adquirido en las fases precedentes (Dirgeyasa, 2016).

La metodología basada en los géneros se destaca por ser una metodología que permite el aprendizaje de la lengua a través de la producción e interpretación de textos en contextos sociales reales e identificables por el estudiantado (Alexopoulou, 2010). Así, el aprendiz consigue identificar los modos en los que los individuos emplean la lengua con objetivos comunicativos determinados y en situaciones de comunicación concreta y puede, del mismo modo, utilizar ese conocimiento al estar expuesto a esas circunstancias (Hyland, 2003). En otras palabras, se trata de una metodología cuya finalidad es que, a través de los géneros discursivos, los aprendices interioricen las convenciones socioculturales, estructurales y lingüísticas que son necesarias para que sea posible el éxito comunicativo.

Para una aplicación óptima de esta metodología, es necesario, en primer lugar, ser conscientes de las necesidades comunicativas de los estudiantes, en este caso, los universitarios (Gardner \& Nesi, 2013). A continuación, sería el momento de llevar a cabo una selección de aquellos géneros discursivos a los que van a estar expuestos: tanto los géneros académicos comunes, como aquellos propios de un área en concreto. En este punto, será necesario deconstruir los textos para identificar las particularidades lingüísticas, socio- 
lingüísticas y pragmático-discursivas de los textos de dicho género. Es importante que se lleve una gradación de la dificultad con la que se exponen los géneros, por lo que se optará por trabajar, en primer lugar, aquellos que no solo entrañen menor dificultad, sino que sean, también, aquellos con los que los aprendices están más familiarizados. En último lugar, será momento de diseñar la tarea de aprendizaje de acuerdo con los objetivos y los contenidos propios del contexto en el que se produzca (Boillos, 2017). No hay que olvidar tampoco la importancia de exponer a los estudiantes a numerosos ejemplos para que puedan emular sus características antes de dar paso al trabajo independiente (Martin \& Rose, 2012).

Parece, en este punto, importante destacar que, gracias a este modo de abordar la enseñanza, los estudiantes pueden ser conscientes de cuáles son sus conocimientos previos en torno a ese género o identificar los conocimientos previos generales que pueden resultarles de utilidad para acometer el reto que se les plantea. Esto favorece también el trabajo interdisciplinar y la conexión de saberes (Zayas, 2012). De la misma manera, aplicar este enfoque implica destacar la labor del docente como guía del proceso de aprendizaje y como agente encargado de elicitar los saberes del alumnado (Rojas et al., 2016).

Por último, y no por ello menos importante, el enfoque basado en los géneros que se sigue en esta propuesta privilegia el trabajo autónomo del aprendiz. Una de las competencias más demandadas en el ámbito laboral y que, como consecuencia, está en el punto de mira de la formación universitaria, es la competencia para el trabajo autónomo. Esta competencia busca, por medio de prácticas de enseñanza controladas, que el estudiante desarrolle su habilidad para razonar, buscar, aplicar conocimiento, revisar, etc. Es decir, se trata de generar ocasiones de aprendizaje en las que los estudiantes identifiquen de qué recursos pueden echar mano para avanzar en su conocimiento (Rué, 2009). Así, este enfoque va acorde a estos principios y, además de elicitar el conocimiento previo del que disponen, dota al estudiante de ejemplos para poder reconocer las situaciones de comunicación y para encarar las demandas comunicativas a las que va a enfrentarse (Boillos, 2017). Por todo ello, no sorprende que se trate de un enfoque que se esté empleando en diversas universidades para el trabajo de la escritura académica (Moyano, 2010).

\section{PROPUESTA DIDÁCTICA}

A continuación, se presenta una descripción de la propuesta para la formación en escritura académica en euskera y para dar respuesta a las demandas previamente expuestas, que lleva por título Oinarrizko idazketa akademikoa garatzeko eskuliburua ${ }^{1}$ (Bereziartua \& Boillos, 2018). Para ello, en primer lugar, se ofrece una presentación del contexto en el que surge. En segundo lugar, se traza la propuesta de manera general para, finalmente, ofrecer descripcio1. Manual para el desarrollo de la escritura académica básica. 


\subsection{CONTEXTO}

A pesar de que esta propuesta pudiera orientarse a otros contextos educativos, parece relevante indicar que es una herramienta creada ad hoc para una asignatura destinada al desarrollo de la competencia comunicativa de los estudiantes que se imparte en euskera en los grados de Educación Infantil y Educación Primaria en facultades de educación de la Comunidad Autónoma Vasca (en adelante, CAV). Se trata, por tanto, de un contexto sociolingüístico bi/plurilingüe en el que conviven las dos lenguas oficiales, el euskera y el castellano (ver Figura 1), y una lengua extranjera que suele ser, mayoritariamente, el inglés.

Figura 1

Competencia Lingüística Según Territorio

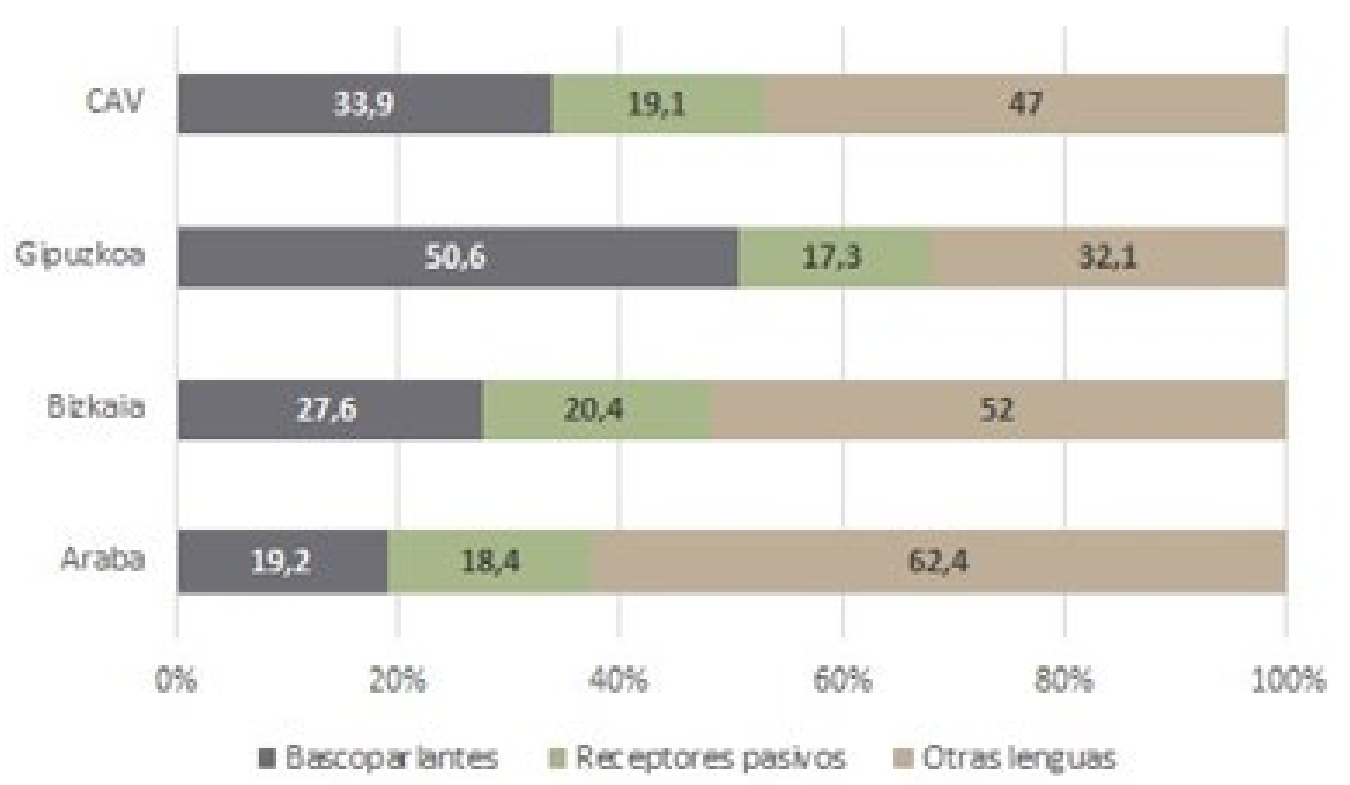

Fuente: VI. Encuesta sociolingüística-2016, pp. 4-5 (Gobierno Vasco, 2016).

Tal y como se puede observar, según los datos de la última encuesta sociolingüística llevada a cabo por el Gobierno Vasco (2016) el 33,9\% de la población de la CAV habla euskera y el 19,1\% es receptor pasivo de esta lengua.

El alumnado actual de esta asignatura, ha cursado estudios preuniversitarios en un modelo educativo bilingüe. Tal como explica Cenoz (2009), el euskera ya se empleaba en el ámbito educativo antes de la Ley de la Normalización del Euskera aprobada en el año 1982, pero dicha ley definió los modelos bilingües de educación en la CAV. El Decreto de Bilingüismo 138/1983 (Boletín Oficial del País Vasco [BOPV] 108/19-7-1983) estableció tres modelos para la escolarización: A, B y D (no existe la letra C en euskera); aunque también existe el modelo $\mathrm{X}$, en el que no hay presencia del euskera. La Tabla 1 muestra las cifras del alumnado matriculado en enseñanzas de régimen general no universitarias en la CAV por territorio histórico y nivel de enseñanza, según titularidad del centro y modelo lingüístico en el curso 2018-2019. 
Como puede verse en la Tabla 1, el modelo D, con un 75\% de las materias en euskera y un $25 \%$ en castellano y lengua extranjera (normalmente, inglés), es el que se imparte mayoritariamente.

Table 1

Porcentaje de alumnado matriculado según etapa educativa y modelo lingüístico en el curso 2017-2018.

\begin{tabular}{llll}
\hline & Modelos A-X & Modelo B & Modelo D \\
\hline Educación Infantil & $3.4 \%$ & $16.3 \%$ & $80.3 \%$ \\
Educación Primaria & $4.7 \%$ & $20.6 \%$ & $74.7 \%$ \\
Educación Secundaria Obligatoria & $8 \%$ & $23.6 \%$ & $68.4 \%$ \\
Bachiller & $31 \%$ & $5 \%$ & $64 \%$ \\
\hline
\end{tabular}

Fuente: Adaptado de Instituto Vasco de Estadística [Eustat] (2019).

En vista de estos resultados, se asume que aquellos estudiantes que optan por matricularse en los grados de Educación Infantil y Educación Primaria en euskera o en la modalidad trilingüe han realizado su formación previa en euskera. Así, y de acuerdo con el Decreto 47/2012 (BOPV, 2012), se podría considerar que el estudiante tiene un dominio equivalente a un B2 en esta lengua. No obstante, como se ha venido apuntando, la formación recibida en estas lenguas no garantiza el dominio para comunicarse en un contexto discursivo, el académico, que hasta ese momento les es desconocido.

De manera paralela, el origen de las actuales necesidades formativas podría vincularse a la históricamente reciente estandarización del euskera. El idioma euskera se estandarizó en el año 1968 (Euskaltzaindia, 1978) y, progresivamente, se ha ido haciendo camino en diferentes ámbitos de uso (medios de comunicación, sistema educativo...). Junto con la históricamente reciente estandarización del euskera se encuentra la también reciente creación de la Universidad del País Vasco en el año 1980, el mayor centro público de la CAV junto con otras universidades privadas como la de Deusto o la de Mondragón, y, con ella, la utilización del euskera en el ámbito académico. Todas estas razones explican la brevedad de la tradición de la producción en euskera en el contexto académico y la consecuente escasez de recursos destinados a la formación del alumnado para tales fines.

Sin embargo, en las últimas décadas, ha proliferado la producción de textos académicos en esta lengua. La base de datos de la comunidad científica vasca (Inguma, 2020) recoge la producción académica en euskera en su totalidad y, a fecha de junio de 2020, tiene constancia de numerosos textos académicos de diferentes géneros textuales: 803 tesis doctorales, 2.607 libros, 16.451 artículos, 93 proyectos de investigación, 5.766 menciones, capítulos y prefacios, entre otros. Estos resultados muestran, por tanto, que la producción académica en euskera está emergiendo mientras que los recursos destinados a la formación no responden a las demandas diagnosticadas. La propuesta que aquí se presenta se trataría, por tanto, de una contribución a esta necesidad. 


\subsection{DESCRIPCIÓN GENERAL DE LA PROPUESTA}

Tal como se ha mencionado, es necesario ser consciente, en primera instancia, de las necesidades comunicativas que van a tener los estudiantes. Cuando estos ingresan en la universidad, se ven expuestos a gestionar y crear textos de corte académico, desde monografías hasta trabajos de fin de grado. Así, en vista de los géneros discursivos a los que con mayor frecuencia van a estar expuestos los estudiantes a lo largo de su formación (Gardner \& Nesi, 2013), esta propuesta toma como base el género artículo científico. Se trata este de un género con el que van a estar familiarizados desde sus primeros días en el mundo académico y que, por extensión, será identificable por parte de los estudiantes (Alexopoulou, 2010).

De acuerdo con las premisas de la Escuela de Sydney y de acuerdo con la primera fase que se establece para el trabajo de los géneros, el modelaje, en este manual se presentan, en primera instancia, dinámicas para la identificación del género que se va a trabajar y para que puedan familiarizarse con él. Para ello, en primer lugar, se ha llevado a cabo una selección de artículos publicados en revistas de indexadas en el ámbito de la Educación que ayudan a garantizar el rigor científico de los mismos y se han deconstruido dichos textos en sus partes prototípicas.

En segundo lugar, se ha procedido al diseño de actividades de diferentes grados de complejidad para cada una de las partes, con el objetivo de que el alumnado cree su propio conocimiento de una manera gradual e inductiva. Se trata de la fase de construcción en la que el alumnado puede jugar con los textos para interiorizar sus características. En estas actividades, de manera paralela, la labor del docente será la de guiar al alumnado en todo el proceso de aprendizaje.

La estructura general de la propuesta se divide en dos partes. En la primera parte, se presentan las actividades prácticas para el alumnado junto con los materiales necesarios para su realización, así como información complementaria que pueda ser de ayuda. En la segunda parte, se dan las claves de dichas actividades, tanto para el profesorado encargado de aplicar esta propuesta como para el alumnado que quiera trabajar de manera autónoma. Tanto las actividades como las explicaciones y los materiales a los que van a estar expuestos los estudiantes están en euskera.

En esta fase las actividades se han dividido de acuerdo con las partes identificadas por la bibiografía como partes prototípicas de este género discursivo: título, abstract, palabras clave, introducción, marco teórico, conclusiones y referencias bibliográficas (Sánchez, 2011). Las partes correspondientes a la metodología y los resultados no se han incluido en la propuesta debido a las variaciones existentes entre diferentes disciplinas y a las variaciones, también, en función de la tipología de investigación llevada a cabo. Asimismo, antes de empezar con las actividades sobre cada parte del artículo científico, se incluyen varias actividades que buscan una primera aproximación a los géneros académicos de manera general. En la Tabla 2, se desglosan los apartados y los contenidos que se abordan en cada uno de ellos. 
Tabla 2

Distribución de la Propuesta Didáctica por Apartados

y Contenidos

\begin{tabular}{ll}
\hline Apartado & Contenido \\
\hline Ejercicios & Identificación de los géneros académicos \\
introductorios & $\begin{array}{l}\text { Identificación de las características prototípicas de cada género } \\
\text { Estrategias para la búsqueda de textos científicos }\end{array}$ \\
\hline Título & Identificación de las características de los títulos de trabajos científicos \\
\hline Abstract & Reconocimiento de la función y composición de los abstract \\
& Creación de abstract \\
\hline Palabras clave & Definición de las palabras clave \\
& Creación y selección de palabras clave \\
\hline Introducción & Caracterización de la introducción \\
& Identificación de las partes de una introducción \\
\hline Marco teórico & Identificación de las características y función del marco teórico \\
& Identificación de las características y función del estado de la cuestión \\
& Distinción entre el marco teórico y el estado de la cuestión \\
& Organización de los contenidos del marco teórico y del estado de la cuestión \\
& Recursos para la identificación del plagio \\
& Técnicas para la paráfrasis y la citación \\
\hline Conclusiones & Caracterización del apartado de las conclusiones \\
& Identificación de las partes del apartado de conclusiones \\
\hline bibliográficas & $\begin{array}{l}\text { Sistemas de referenciación } \\
\text { Características del sistema de citación APA } \\
\text { Identificación de la información contenida en una referencia bibliográfica }\end{array}$ \\
\hline
\end{tabular}

Fuente: Elaboración propia.

En la segunda parte de este manual se dan las claves o soluciones de las actividades prácticas. Estas sirven para el trabajo autónomo y como guía para el profesorado. La información sobre las claves se presenta clasificada en los siguientes apartados: descripción breve de la actividad, el tiempo requerido para realizar la actividad, los objetivos de la actividad, los recursos complementarios necesarios, materiales empleados, fuente y respuestas correctas. En la Tabla 3, se describen de manera pormenorizada los contenidos de cada apartado. 
Tabla 3

Información Proporcionada en las Claves de las Actividades Prácticas

\begin{tabular}{|c|c|}
\hline Apartado & Información que se proporciona \\
\hline $\begin{array}{l}\text { Descripción breve } \\
\text { de la actividad }\end{array}$ & $\begin{array}{l}\text { Se explica qué tienen que hacer los alumnos en cada una de las } \\
\text { actividades. } \\
\text { Ejemplo: } \\
\text { 5.1.3. CONOCER LOS GÉNEROS ACADÉMICOS } \\
\text { Por una parte, se proporcionarán a los estudiantes textos de diversos } \\
\text { géneros discursivos; por otra, se facilitará un listado de géneros. A cada } \\
\text { texto tendrán que asignarle un género discursivo. } \\
\text { Una vez realizada esta tarea, tendrán que encontrar otro ejemplo de cada } \\
\text { uno de los géneros. }\end{array}$ \\
\hline $\begin{array}{l}\text { Tiempo requerido } \\
\text { para realizar la } \\
\text { actividad }\end{array}$ & $\begin{array}{l}\text { Se dan orientaciones en torno al tiempo previsto para la realización de la } \\
\text { tarea. } \\
\text { Ejemplo: } \\
\text { 15' }\end{array}$ \\
\hline Objetivos & $\begin{array}{l}\text { Se presentan los objetivos que se van a alcanzar con dicha actividad. } \\
\text { Ejemplo: } \\
\text { Identificar los géneros discursivos. }\end{array}$ \\
\hline $\begin{array}{l}\text { Recursos } \\
\text { complementarios }\end{array}$ & $\begin{array}{l}\text { Se indica qué materiales o recursos, además del manual, son necesarios } \\
\text { para realizar la actividad (dispositivo con conexión a Internet, dispositivos } \\
\text { digitales, cronómetro...). } \\
\text { Ejemplo: } \\
\text { Una herramienta para conectarse a Internet. }\end{array}$ \\
\hline $\begin{array}{l}\text { Materiales } \\
\text { empleados }\end{array}$ & $\begin{array}{l}\text { Se presentan las referencias de los materiales que se han empleado } \\
\text { para diseñar la actividad (referencias bibliográficas de los documentos } \\
\text { científicos, hipervínculos, link a las bases de datos, direcciones web de las } \\
\text { revistas científicas, etc.). } \\
\text { Ejemplo: } \\
\text { Guerenabarrena, M. (2016). EAEn 4. DBHko ikasleen euskara erabileran } \\
\text { eragiten duten faktoreak (doktorego-tesia). Euskal Hizkuntza eta } \\
\text { Komunikazioa saila, UPV/EHU. http://hdl.handle.net/10810/18877 } \\
\text { Celorio, G. eta López de Muniain, A. (konp.) (2006). Garapenerako } \\
\text { Hezkuntzari buruzko III. Kongresua: Hezkuntza eraldatzailea } \\
\text { globalizazioaren erronken aurrean. Gasteiz, Hegoa. http://alboan.efaber.net/ } \\
\text { ebooks/0000/0054/Hegoa_Aktak_EU.pdf } \\
\text { Lekaroz, G. (2006). Antzinako baztanera:XVIII. mendeko prediku } \\
\text { argitaragabe bat. Fontes linguae vasconum: Studia et documenta, 38 (101), } \\
\text { 69-94. http://www.navarra.es/NR/rdonlyres/590688AB-45C1-4F0A-88B7- } \\
\text { 848AFC942B3D/254790/FOLI0101000000690094.pdf } \\
\text { Jiménez, L. (2015). Joko sortzailea Haur Hezkuntzan (Gradu Amaierako } \\
\text { Lana). Bilboko Irakasleen Unibertsitate Eskola, UPV/EHU. https://addi. } \\
\text { ehu.es/bitstream/handle/10810/17669/Joko\%20sortzailea\%20Haur\%20 } \\
\text { Hezkuntzan.pdf?sequence=3\&isAllowed=y }\end{array}$ \\
\hline Fuente & $\begin{array}{l}\text { En los casos en los que la actividad se ha tomado o adaptado de otros } \\
\text { materiales, se deja constancia de ello en este apartado para que el usuario } \\
\text { pueda acceder directamente a la fuente original. }\end{array}$ \\
\hline Respuestas correctas & $\begin{array}{l}\text { Se proporciona la respuesta correcta en aquellos casos en los que solo } \\
\text { exista una respuesta posible y se proporciona una respuesta posible cuando } \\
\text { son posibles varias respuestas correctas. } \\
\text { Ejemplo: } \\
\text { a: } 3 \text {, tesis doctoral. } \\
\text { b: } 1 \text {, acta. } \\
\text { c: } 2 \text {, artículo científico. } \\
\text { d: } 4 \text {, Trabajo de final de grado (TFG). }\end{array}$ \\
\hline
\end{tabular}

Fuente: Elaboración propia. 
Después de las dos partes fundamentales de la propuesta, llega el momento de la fase de construcción independiente. El alumnado ya ha podido interiorizar las características del género discursivo y es momento de que aborde la escritura de un texto de esta naturaleza de manera autónoma. Para ayudar en esta labor, se presenta una checklist o lista de comprobación (ver Anexo I). El objetivo de esta herramienta es facilitar al usuario de la propuesta un instrumento para hacer una revisión final del texto producido. Al crear un texto académico es importante intentar cuidar todos los aspectos durante el proceso de creación, pero tan importante como eso es hacer una lectura global una vez finalizado el texto. Por dicho motivo, se ofrece una lista de interrogantes con la que el estudiante podrá comprobar la calidad de su trabajo. Esta herramienta favorece, además, el desarrollo de las habilidades para el aprendizaje autónomo de los aprendices (Boillos, 2017).

A continuación, se presentan, a modo de ejemplo, algunos de los indicadores para comprobar la pertinencia del título del trabajo que hayan creado:

- El título responde a las características de los títulos de los trabajos académicos;

- El título plantea con claridad el tema y los objetivos del trabajo.

Y he aquí otros indicadores acerca de las palabras clave que hayan seleccionado:

- La cantidad de palabras clave responde a la petición de la revista;

- Las palabras clave utilizadas reflejan el contenido del texto completo;

- Las palabras clave seleccionadas se encuentran en el Tesauro de la UNESCO.

Nótese que estos se han diseñado de acuerdo con los contenidos del manual. De ahí que sea necesario realizar previamente las actividades del manual para poder comprender la terminología empleada en la checklist.

Finalmente, se incluye una lista de bibliografía complementaria donde se facilita información sobre manuales, artículos y páginas web que pueden ser de utilidad para seguir profundizando en la materia.

\subsection{DESCRIPCIÓN DE ALGUNAS ACTIVIDADES}

Las premisas en las que se basa el diseño de las actividades son las siguientes: añadir dificultad gradualmente a las actividades y utilizar métodos inductivos con el fin de activar los conocimientos previos del alumnado (Zayas, 2012) y con el fin, también, de proporcionar ejemplos para que puedan ir construyendo su propio conocimiento. A continuación, se presentan algunos ejemplos de actividades y su fundamentación de acuerdo con las ideas previamente expuestas.

Uno de los puntos que se han mencionado en el marco teórico es que hay que dar a conocer al alumnado las características discursivas de los textos que se producen en una comunidad. Para ello, y de acuerdo con las premisas para diseñar las actividades, se propone la secuencia de actividades de la Tabla 4. 
Tabla 4

Síntesis de las Actividades 4.1.1, 4.1.3 y 4.1.4

\begin{tabular}{|c|c|c|}
\hline $\begin{array}{l}\text { N. }{ }^{\circ} \text { de } \\
\text { actividad }\end{array}$ & Descripción & Objetivos \\
\hline 4.1 .1 & $\begin{array}{l}\text { Identificar géneros académicos } \\
\text { - Se ponen a disposición del alumnado fragmentos de } \\
\text { textos de varios géneros: la entrada de un diccionario } \\
\text { enciclopédico, la sinopsis de una novela literaria, una } \\
\text { noticia de periódico, una entrada de wikipedia, la parte } \\
\text { de un artículo científico y una crítica literaria. } \\
\text { - Tienen que identificar el texto académico y } \\
\text { argumentar sus respuestas. }\end{array}$ & $\begin{array}{l}\text { - Activar los conocimientos } \\
\text { previos } \\
\text { - Identificar las características } \\
\text { generales de los textos } \\
\text { académicos }\end{array}$ \\
\hline 4.1 .3 & $\begin{array}{l}\text { Conocer géneros académicos } \\
\text { - Por un lado, se presenta una lista de varios géneros } \\
\text { académicos: Trabajo de Fin de Grado, tesis doctoral, } \\
\text { acta de un congreso y artículo científico. } \\
\text { - Por otro lado, se dan los enlaces a textos electrónicos } \\
\text { de los géneros académicos presentados, pero de } \\
\text { manera desordenada. } \\
\text { - El alumnado ha de relacionar cada documento con } \\
\text { un género académico. }\end{array}$ & $\begin{array}{l}\text { - Activar los conocimientos } \\
\text { previos } \\
\text { - Conocer varios géneros } \\
\text { académicos }\end{array}$ \\
\hline 4.1.4 & $\begin{array}{l}\text { Conocer las características de los géneros académicos } \\
\text { - Al hilo de los géneros académicos identificados en } \\
\text { la actividad anterior, el alumnado ha de completar } \\
\text { una tabla con la siguiente información sobre cada } \\
\text { género: extensión, espacio donde se publica, a quién } \\
\text { va destinado, entre otros. }\end{array}$ & $\begin{array}{l}\text { - Familiarizarse con varios } \\
\text { géneros académicos } \\
\text { - Conocer las características de } \\
\text { varios géneros académicos }\end{array}$ \\
\hline
\end{tabular}

Fuente: Bereziartua y Boillos (2018).

Las actividades prácticas propuestas en la Tabla 4 presentan diferentes grados de complejidad. De acuerdo con la taxonomía revisada de Bloom (Anderson y Krathwohl, 2001), en esta propuesta pueden encontrarse actividades que exigen habilidades de pensamiento de orden inferior (recordar y comprender) como actividades que exigen habilidades de pensamiento de orden superior (aplicar, analizar, evaluar y crear). En la secuencia anterior se puede apreciar que todas las actividades responden a un nivel de complejidad de orden inferior. La primera actividad se situaría en el primer grado de complejidad (recordar) y las dos actividades siguientes en el segundo grado de dificultad (comprender).

Esta secuencia de actividades favorece que el estudiante identifique que la escritura que se produce en el ámbito académico tiene unas particularidades que la distinguen de las prácticas escritoras llevadas a cabo hasta el momento (Carlino, 2013). Así también, van a identificar que los géneros en concreto que se producen en este ámbito tienen unos objetivos determinados y unas características concretas que están determinadas por el contexto social e histórico en el que se producen (Gardner \& Nesi, 2013).

En la Tabla 5, se muestra una secuencia de actividades donde el abanico de graduación de la complejidad es más amplio: la primera actividad se situaría en el segundo grado de complejidad (comprender) dentro del nivel de complejidad de orden inferior; la segunda actividad responde al tercer grado (aplicar) dentro del nivel de complejidad de orden superior; y la tercera actividad exige el mayor grado de complejidad (crear) (Anderson \& Krathwohl, 2001). 
Tabla 5

Síntesis de las Actividades 4.3.2, 4.3.4 y 4.3.9

\begin{tabular}{|c|c|c|}
\hline N..$^{\circ}$ de actividad & Descripción & Objetivos \\
\hline 4.3.2 & $\begin{array}{l}\text { Identificar las subsecciones de un abstract } \\
\text { - Se enseñan los ejemplos de dos abstracts } \\
\text { y se le pide al alumnado que identifique las } \\
\text { subsecciones de estos. }\end{array}$ & $\begin{array}{l}\text { - Activar conocimientos previos } \\
\text { - Identificar las subsecciones } \\
\text { que componen un abstract } \\
\text { - Conocer la estructura de un } \\
\text { abstract } \\
\text { - Familiarizarse con la lógica de } \\
\text { los textos académicos }\end{array}$ \\
\hline 4.3.4 & $\begin{array}{l}\text { Ordenar las subsecciones de un abstract } \\
\text { - Se muestran las frases que componen } \\
\text { un abstract de manera individual y } \\
\text { desorganizada. } \\
\text { - El alumnado ha de ordenar las frases. }\end{array}$ & $\begin{array}{l}\text { - Familiarizarse con el } \\
\text { subgénero del abstract } \\
\text { - Interiorizar las subsecciones de } \\
\text { un abstract }\end{array}$ \\
\hline 4.3 .9 & $\begin{array}{l}\text { Crear nuestro abstract } \\
\text { - Se le facilita al alumnado la información } \\
\text { necesaria para poder crear un abstract. Por } \\
\text { ejemplo: } \\
\text { - Tema: La alimentación y el desarrollo motor } \\
\text { de los niños. } \\
\text { - Objetivo: Ver si la alimentación del } \\
\text { alumnado de Educación Primaria influye en } \\
\text { su desarrollo motor. } \\
\text { - Metodología: Muestra e instrumentos para } \\
\text { la recogida de datos (inventar). } \\
\text { - Resultados y conclusiones: (inventar). } \\
\text { - El alumnado se agrupa de dos en dos y cada } \\
\text { pareja crea un abstract. }\end{array}$ & $\begin{array}{l}\text { - Activar los conocimientos } \\
\text { previos } \\
\text { - Activar la capacidad para } \\
\text { pensar de manera académica } \\
\text { - Aplicar el conocimiento } \\
\text { adquirido en actividades previas } \\
\text { - Crear un producto de corte } \\
\text { académico }\end{array}$ \\
\hline
\end{tabular}

Fuente: Bereziartua y Boillos (2018).

\subsection{BENEFICIOS ESPERADOS DE LA PROPUESTA}

Como se ha podido observar a partir de la aplicación de este material en el contexto de estudio, esta secuencia de tareas va en consonancia con los planteamientos previamente expuestos de Castelló (2009), quien apuntaba a la necesidad no solo de identificar la situación de comunicación, sino de poner en marcha las tareas que requiere el proceso de composición del texto. En este caso en concreto, se busca estimular la búsqueda de información o la reorganización de ideas, entre otras fases, y favorecer el contacto con fragmentos de texto propios del área del saber del que van a formar parte los estudiantes. Es decir, las tres dimensiones a las que hacía mención la autora.

Se ha observado, además, que esta ejercitación puede favorecer el desarrollo de las habilidades propias de los escritores expertos (Scardamalia \& Bereiter, 1992) en tanto que posibilita la autorregulación del aprendizaje y la toma de conciencia de los procesos que intervienen en el proceso de composición del texto. Otro valor añadido de estas actividades es la conexión de saberes (Zayas, 2012). Prueba de ello es la actividad del abstract con la que ejercitan, en un texto breve e identificable, un conocimiento que será aplicable, posteriormente, a un texto más extenso - y, quizá, menos conocido - como es el artículo científico. En este sentido, se puede vaticinar que el incremento 
progresivo de dificultad que se ha programado en las actividades dotará al alumnado de estrategias para la escritura que le serán de utilidad a lo largo de formación universitaria.

Se espera, además, que la modalidad de trabajo que se propone facilite el abordar las carencias escritoras de manera autónoma. El tener acceso tanto a las respuestas como a materiales complementarios hará innecesaria una intervención continua del docente quien pasa a tener un rol de guía en este proceso. Es importante destacar que esto es útil especialmente para los docentes no versados en esta materia. Por último, la checklist final busca que el alumnado pueda ser consciente de sus conocimientos ya adquiridos y pueda autoevaluarse o, en algunos casos, coevaluarse.

En definitiva, este proceso en el que se desmiembra el texto desde las premisas del enfoque basado en los géneros (Rose, 2018) tiene como finalidad dar respuesta a las demandas que se han diagnosticado en el ámbito académico en relación con las habilidades comunicativas escritas de los estudiantes. Unas demandas aún más evidentes en contextos como el que es objeto de estudio en el que se producen textos en una lengua minoritaria cuyo discurso académico está en fase aún de desarrollo.

\section{CONCLUSIONES}

No existe duda al afirmar que parte del éxito del alumnado en la etapa universitaria depende de su capacidad para desenvolverse en las situaciones de comunicación que se producen dentro del medio académico. De ahí la importancia de generar propuestas didácticas que tengan como objetivo familiarizar al estudiante con los géneros discursivos propios de este ámbito desde los inicios de su formación universitaria. Esto es más significativo, si cabe, en contextos en los que esas producciones escritas se producen en más de una lengua. Sin olvidar la importancia que tiene cuando una de estas es una lengua minoritaria.

En este trabajo, concretamente, se ha descrito una propuesta para trabajar el género artículo científico con estudiantes de perfil bilingüe (euskera-castellano) que están cursando sus estudios universitarios en euskera. La propuesta, fundamentada en el enfoque basado en los géneros, aborda cada parte del género artículo científico de manera desglosada y con un nivel de dificultad creciente. Asimismo, las actividades van acompañadas de una clave de respuestas que favorecen el aprendizaje autónomo y que buscan ayudar a los docentes en la compleja tarea que supone formar en las habilidades escritas.

Como futuras fases en este desafío, sería de interés llevar a cabo estudios de investigación en acción que permitan medir los beneficios que conlleva la aplicación de este material en el contexto educación. La finalidad última será medir el impacto a largo plazo que propuestas como la aquí presentada tienen en el proceso de alfabetización académica de los estudiantes de grado. Es decir, comprobar si este tipo de ejercicios favorecen la adquisición de las particularidades de la escritura propia de este género discursivo y, como consecuencia, mejora también su competencia escritora en el contexto 
universitario. En este sentido, sería clave medir si este proceso de aprender a escribir tiene como resultado también, como apuntaba Capomagi (2013), una mejor capacidad para aprender en el resto de las materias.

\section{REFERENCIAS}

Aguilar, P. (2017). Una propuesta de géneros discursivos escritos del ámbito universitario, jurídico y chileno, orientada a la alfabetización académica de estudiantes de derecho. Perfiles Educativos, 39(155), 179-192. https://doi. org/10.22201/iisue.24486167e.2017.155.58063

Alexopoulou, A. (2010). El enfoque basado en los géneros textuales y la evaluación de la competencia discursiva. In J. de Santiago, H. Bongaerts, J. J. Sánchez, \& M. Seseña (Coords.), Actas del XXI Congreso Internacional de ASELE (pp. 97-110). Universidad de Salamanca. https://doi.org/10.13140/2.1.1015.4565

Anderson, L. W., \& Krathwohl, D. R. (2001). A taxonomy for learning, teaching, and assessing: A revision of Bloom's taxonomy of educational objectives. Longman.

Bajtín, M. (1979). Estética de la creación verbal. Siglo X.

Bereziartua, G., \& Boillos, M. M. (2018). Oinarrizko idazketa akademikoa garatzeko eskuliburua. UPV/EHUko ikasmaterialen sare-argitalpenak. https://ikasmaterialak.ehu.eus/hezkuntza/ oinarrizko-idazketa-akademikoa-garatzeko-eskuliburua

Boillos, M. M. (2017). Propuesta para la enseñanza de la escritura a través de los géneros en la Educación Secundaria. Didáctica. Lengua y Literatura, 29, 11-28. https://doi.org/10.5209/DIDA.57127

Boillos, M. M. (2018). La autopercepción de las habilidades escritoras en el inicio de la etapa universitaria. Ensayos: Revista de la Facultad de Educación de Albacete, 33(2), 149-160. https://revista.uclm.es/index.php/ensayos/article/ view/1842

Boletín Oficial del País Vasco (1983). DECRETO 138/1983, de 11 de julio, del Departamento de Educación y Cultura, por el que se regula el uso de las lenguas oficiales en la enseñanza no universitaria en el País Vasco. https://www.euskadi.eus/ y22-bopv/es/bopv2/datos/1983/07/8301433a.shtml

Boletín Oficial del País Vasco (2012). DECRETO 47/2012, de 3 de abril, de reconocimiento de los estudios oficiales realizados en euskera y de exención de la acreditación con títulos y certificaciones lingüisticas en euskera. http://www.euskadi. eus/web01-a2larri/es/contenidos/decreto/bopv201201701/es_def/index.shtml

Capomagi, D. (2013). La escritura académica en el aula universitaria. Revista de Educación y Desarrollo, 25, 29-40. http://www.cucs.udg.mx/revistas/edu_ desarrollo/anteriores/25/025_Capomagi.pdf

Carlino, P. (2002). ¿Quién debe ocuparse de enseñar a leer y a escribir en la universidad? Lectura y Vida, 23(1), 6-14.

Carlino, P. (2005). Escribir, leer y aprender en la universidad: Una introducción a la alfabetización académica. Fondo de Cultura Económica, FCE.

Carlino, P. (2013). Alfabetización académica diez años después. Revista Mexicana de Investigación Educativa, 18(57), 355-381.

Carlino, P., Iglesias, P., \& Laxalt, I. (2013). Concepciones y prácticas 
declaradas de profesores terciarios en torno al leer y escribir en las asignaturas. REDU - Revista de Docencia Universitaria, 11(1), 105-136. https://doi.org/10.4995/ redu.2013.5594

Carlino, P., \& Martínez, S. (Coord.). (2009). La lectura y la escritura: Un asunto de todos/as. Educo.

Castelló, M. (2009). Escribir trabajos de investigación con alumnos de grado. Textos de Didáctica de la Lengua y de la Literatura, 20, 21-29.

Cenoz,J. (2009). Towards multilingual education: Basque educational research from an international perspective. Multilingual Matters.

Dirgeyasa, I. W. (2016). Genre-based approach: What and how to teach and to learn writing. English Language Teaching, 9(9). http://dx.doi.org/10.5539/elt. v9n9p45

Euskaltzaindia. (1978). Euskara Batua. Euskera Aldizkaria, 24, 441-477.

Ezeiza, J. (2008). Las competencias comunicativolingüísticas de tipo disciplinar: Perfiles profesionales y recursos para su desarrollo en el nuevo EEES [Espacio Europeo de Educación Superior]. Actas del XXXVII Simposio Internacional de la Sociedad Española de Lingüística (pp.131-144). http://www.ehu.eus/PAT/compe/ lanak/Ezeiza_2.pdf

Gardner, S., \& Nesi, H. (2013). A classification of genre families in university student writing. Applied Linguistics, 34(1), 25-52. https://doi.org/10.1093/ applin/ams024

Gobierno Vasco - Eusko Jaurlaritza. (2016). VI. Inkesta Soziolinguistikoa. https://www.irekia.euskadi.eus/uploads/attachments/9954/NI_INK_SOZLG-EH_eus. pdf?1499236557

Guzmán-Simón, F., \& García-Jiménez, E. (2017). La alfabetización académica de los futuros maestros. Un estudio comparativo en varias universidades españolas. Revista de Investigación Educativa, 35(2), 317-335. http://dx.doi. org/10.6018/rie.35.2.246011

Hyland, K. (2003). Genre-based pedagogies: A social response to process. Journal of Second Language Writing, 12(1), 17-29. http://dx.doi.org/10.1016/ S1060-3743(02)00124-8

Inguma. (2020). Datos de Inguma. Inguma. Base de datos de la comunidad científica vasca. http://www.inguma.eus/ingumako-datuak

Instituto Vasco de Estadística. (2019). Alumnado matriculado en enseñanzas de régimen general no universitarias en la C.A. de Euskadi por ámbitos territoriales, según nivel de enseñanza y modelo lingüístico. 2018-19. http://www.eustat.eus/ elementos/ele0000100/Alumnado_matriculado_en_ensenanzas_de_regimen_ general_no_universitarias_en_la_CA_de_Euskadi_por_ambitos_territoriales_segun_ nivel_de_ensenanza_y_modelo_linguistico/tbl0000106_c.html

Lea, M. R., \& Street, B. (2006). The 'academic literacies' model: Theory and applications. Theory Into Practice, 45(4), 368-377. https://doi.org/10.1207/ s15430421tip4504_11

Martin, J. R. (1999). Factual writing: Exploring and challenging social reality. Deakin University Press.

Martin, J. R., \& Rose, D. (2012). Learning to write, reading to learn. Equinox.

Moyano, E. (2010). Escritura académica a lo largo de la carrera: Un programa institucional. Signos, 43(74), 465-488. http://dx.doi.org/10.4067/ S0718-09342010000500004 
Prior, P., \& Bilbro, R. (2012). Academic enculturation: Developing literate practices and disciplinary identities. In M. Castelló \& C. Donahue (Eds.), University writing: Selves and texts in academic societies (pp. 20-31). Emerald.

Rojas, I., Olave, G., \& Cisneros, M. (2016). Alfabetización académica y pedagogía de género discursivo en la lingüística sistémico funcional. Una experiencia de trabajo. Signos, 49(S1), 224-246. http://dx.doi.org/10.4067/ S0718-09342016000400011

Rose, D. (2015). New developments in genre-based literacy pedagogy. In C. A. MacArthur, S. Graham, \& J. Fitzgerald (Eds.), Handbook of writing research (pp. 227-242). Guildford.

Rose, D. (2017). Languages of schooling: Embedding literacy learning with genre-based pedagogy. European Journal of Applied Linguistics, 6(1), 59-89. https://doi.org/10.1515/eujal-2017-0008

Rose, D., \& Martin, J. R. (2012). Learning to write, reading to learn: Genre, knowledge and pedagogy in the Sydney School. Equinox.

Rué, J. (2009). El aprendizaje autónomo en Educación Superior. Narcea.

Russell, D. R., \& Cortes, V. (2012). Academic and scientific texts: The same or different communities? In M. Castelló \& C. Donahue (Eds.), University writing. Selves and texts in academic societies (pp. 3-17). Emerald.

Sánchez, A. A. (2011). Manual de redacción académica e investigativa: Cómo escribir, evaluar y publicar artículos. Católica del Norte Fundación Universitaria.

Scardamalia, M., \& Bereiter, C. (1992). Dos modelos explicativos de los procesos de composición escrita. Infancia y Aprendizaje, (15)58, 43-64. https://doi.org /10.1080/02103702.1992.10822332

Swales, J. (1996). Genre analysis. English in academic and research settings. Cambridge University Press.

Werlich, E. (1975). Typologie der texte. Fink.

Zayas, F. (2012). Los géneros discursivos y la enseñanza de la composición escrita. Revista Iberoamericana de Educación, 59, 63-85. https://doi.org/10.35362/ rie590457 
i Universidad del País Vasco/Euskal Herriko Unibertsitatea, Facultad de Educación de Bilbao, España. https: //orcid.org/0000-0001-5546-4724

ii Universidad del País Vasco/Euskal Herriko Unibertsitatea, Facultad De Educación, Filosofía y Antropología, España. https://orcid.org/0000-0003-4987-3315

Toda a correspondência relativa a este artigo deve ser enviada para:

Mari Mar Boillos Pereira

Departamento de Didáctica de la Lengua y de la Literatura

Facultad de Educación de Bilbao - UPV/EHU

Barrio Sarriena $s / n$

48940 Leioa (Bizkaia)

mariadelmar.boillos@ehu.eus

Recebido em 10 de fevereiro de 2020

Aceite para publicação em 25 de julho de 2020 


\title{
Didactic proposal for a first approach to the writing
} of the scientifics papers in Basque

\begin{abstract}
Writing academic texts is one of the first tasks students face when entering university. Starting studies in Higher Education brings the need to learn the forms of oral and written interaction of this new discourse community and, consequently, an academic literacy acquisition process begins. Writing will also be the tool with which the students will show the knowledge learned and the means by which they will be evaluated. This work presents a didactic tool that has the purpose of helping students to carry out, with the help of teachers or autonomously, a first approach to the writing of academic texts in Basque, regardless of the area in which they are receiving their training. It is a didactic proposal that seeks to improve the formation in Higher Education and to respond to the educational demands through a genre-based approach. In this case, the selected genre is the scientific article. The application of this proposal contributes to identify the characteristics of the academic texts and to become familiar with the communicative demands of this type of productions. In parallel, it promotes autonomous learning and the development of useful discursive skills to function in the university community.
\end{abstract}

Keywords: Biographical research; Biographical

interview; Relational dimension; Adult educators. 


\section{Proposta didática para uma primeira abordagem à escrita de um artigo científico em língua basca}

\section{RESUMO}

Produzir textos académicos escritos é uma das primeiras tarefas que os estudantes enfrentam quando entram na universidade. 0 início dos estudos no Ensino Superior traz consigo a necessidade de aprender as formas de interação oral e escrita dessa nova comunidade discursiva e, consequentemente, de iniciar um processo de alfabetização académica. A escrita também será a ferramenta com a qual os alunos mostrarão o conhecimento aprendido e o meio pelo qual serão avaliados. Este trabalho apresenta uma ferramenta didática que visa apoiar os alunos na apropriação, com a ajuda do profesor ou autonomamente, dos fundamentos da escrita académica em basco, independentemente do curso superior que frequentam. Trata-se de uma proposta de material didático que procura aprimorar a formação no Ensino Superior e responder às demandas educacionais por meio de uma abordagem baseada em géneros discursivos. Neste caso, o género selecionado é o artigo científico. A aplicação desta proposta ajuda a identificar as características desses textos académicos e a familiarizar-se com as demandas comunicativas desse tipo de produção. Paralelamente, promove a aprendizagem autónoma e o desenvolvimento de habilidades discursivas úteis para funcionar na comunidade universitária.

Palavras-chave: Alfabetização académica; Géneros discursivos; Ferramenta didática; Ensino Superior. 


\section{ANEXO I.}

\section{Checklist}

[Versión traducida al castellano del euskera.]

\begin{tabular}{|c|c|c|}
\hline Secciones & Puntos que comprobar & \\
\hline \multirow[t]{4}{*}{$\begin{array}{l}\text { PRIMEROS } \\
\text { PASOS }\end{array}$} & $\begin{array}{l}\text { Tengo claro cuál es el género discursivo que voy a escribir: sus } \\
\text { características, dónde se publica, a quién está dirigido, etc. }\end{array}$ & \\
\hline & $\begin{array}{l}\text { Antes de empezar, he tenido en cuenta cuáles son las partes de este } \\
\text { texto. }\end{array}$ & \\
\hline & He tenido en cuenta el formato de la revista. & \\
\hline & He mirado de antemano el número de palabras y de páginas establecido. & \\
\hline \multirow[t]{2}{*}{ TÍTULO } & $\begin{array}{l}\text { El título responde a las características de los títulos de los trabajos } \\
\text { académicos. }\end{array}$ & \\
\hline & El título plantea con claridad el tema y los objetivos del trabajo. & \\
\hline \multirow[t]{3}{*}{ ABSTRACT } & $\begin{array}{l}\text { He tenido en cuenta las características principales de los resúmenes } \\
\text { científicos. }\end{array}$ & \\
\hline & No he cometido ninguno de los errores frecuentes. & \\
\hline & He comprobado que contiene todas las partes de un resumen científico. & \\
\hline \multirow[t]{3}{*}{ PALABRAS CLAVE } & La cantidad de palabras clave responde a la petición de la revista. & \\
\hline & Las palabras clave utilizadas reflejan el contenido del texto completo. & \\
\hline & $\begin{array}{l}\text { Las palabras clave seleccionadas se encuentran en el Tesauro de la } \\
\text { UNESCO. }\end{array}$ & \\
\hline \multirow[t]{8}{*}{$\begin{array}{l}\text { INTRODUCCIÓN } \\
\text { MARCO TEÓRICO }\end{array}$} & $\begin{array}{l}\text { He incluido las partes de una introducción (qué, por qué, para qué y } \\
\text { organización). }\end{array}$ & \\
\hline & $\begin{array}{l}\text { En el inicio de cada uno de los apartados he empleado una fórmula para } \\
\text { que se pueda identificar su contenido. }\end{array}$ & \\
\hline & He buscado las referencias en bases de datos de calidad y rigor científico. & \\
\hline & He aplicado el CRAAP a los textos que he encontrado. & \\
\hline & He diferenciado el marco teórico y el state of art. & \\
\hline & He incluido citas cuando he utilizado las ideas de otras personas. & \\
\hline & He cumplido las normas APA. & \\
\hline & $\begin{array}{l}\text { He utilizado alguna herramienta de detección de plagio para comprobar } \\
\text { la originalidad de mi trabajo. }\end{array}$ & \\
\hline \multirow[t]{2}{*}{ CONCLUSIONES } & He escrito el apartado de conclusiones. & \\
\hline & $\begin{array}{l}\text { En el inicio de cada uno de los apartados he empleado una fórmula para } \\
\text { que se pueda identificar su contenido. }\end{array}$ & \\
\hline \multirow[t]{3}{*}{ REFERENCIAS } & He incluido todos los trabajos citados a lo largo del texto. & \\
\hline & Todas las fuentes seleccionadas son científicas. & \\
\hline & He cumplido las normas APA. & \\
\hline
\end{tabular}

